\title{
Un conseil scientifique à la Société française de médecine périnatale
}

\author{
U. Simeoni \\ (C) Springer-Verlag France 2009
}

La Société française de médecine périnatale a vu la naissance, en 2009, d'un conseil scientifique (CS). Évolution marquante au sein de la société, ce conseil est le résultat d'une réflexion du conseil d'administration, lancée au début de l'année 2008. Le constat était que, certes, la SFMP assurait avec régularité sa mission principale de formation périnatale pluridisciplinaire, mais qu'il devenait probablement nécessaire de préparer l'avenir et de favoriser un renouvellement des idées et des thèmes des congrès, et une ouverture supplémentaire dans les communications libres présentées.

L'objectif de ce CS est triple :

- assurer l'organisation des sessions de communications libres ;

- être une force de proposition thématique pour le congrès annuel de la société ;

- élaborer des avis d'ordre scientifique sur les questions de médecine périnatale qui peuvent émerger de la réflexion des membres de la société ou de sollicitations externes (recommandations formalisées d'experts, questions issues de l'actualité médicale ou sociétale).

Les résumés soumis à la société, en vue d'une communication lors de son congrès, sont désormais confiés au CS, qui est ainsi intervenu dès le congrès de 2009, à Angers. La méthode choisie se veut rigoureuse et objective, tout en visant à permettre au plus grand nombre de participants de présenter une contribution originale, sous une forme orale ou affichée. Les résumés sont rendus anonymes puis évalués par les membres du CS, en fonction de leur spécialité ; l'ensemble des évaluations est revu en séance, de façon à vérifier la pertinence et la cohérence des décisions et à organiser des sessions qui seront désormais de type thématique au congrès. Très peu de propositions ont fait l'objet d'une décision négative, le plus souvent en raison d'une documentation insuffisante du contenu. Un niveau de sélectivité aussi faible peut être critiqué, mais le souci a été surtout de valoriser les contributions, y compris celles

U. Simeoni $(\bowtie)$

Service de médecine néonatale, hôpital de La Conception, AP-HM \& Université de la Méditerranée,

147, boulevard Baille, F-13385 Marseille cedex 05, France e-mail : umberto.simeoni@mail.ap-hm.fr de type empirique, et de permettre la participation et la discussion collégiale.

Les propositions par le CS de thématiques pour les congrès concerneront déjà le congrès de 2011 (le programme de celui de 2010 étant déjà arrêté). Elles porteront sur les tables rondes, les exposés didactiques relevant directement du comité local d'organisation du congrès. Le but sera de « coller » à l'actualité, d'innover autant que possible, tout en respectant l'approche essentiellement pratique et pluridisciplinaire qui fait la richesse des congrès de la société.

Enfin, les questions et les sollicitations d'avis, internes et externes, seront traitées avec une méthode de revue systématique structurée, aboutissant à une position argumentée scientifiquement sur les questions liées à la médecine de la mère et de l'enfant et à ce qui l'entoure, en particulier sur le plan humain. L'intérêt particulier de ce conseil réside, en effet, dans la représentativité périnatale de sa composition.

Le CS se réunira deux fois par an, en liaison avec le conseil d'administration, vis-à-vis duquel il aura un rôle consultatif. Les membres de cette première équipe ${ }^{1}$ ont été cooptés et choisis en fonction de leur compétence scientifique, de leur représentativité et souvent de leur origine nouvelle par rapport aux autres contributeurs au fonctionnement de la société, en respectant l'indispensable équilibre représentant les disciplines de l'obstétrique, de la néonatologie, de l'épidémiologie, de l'anesthésie-réanimation, de la psychologie notamment. Le CS se renouvellera par tiers tous les ans, ce qui permettra régulièrement à de nouvelles motivations de s'exprimer.

Ainsi, la création de ce CS ne signifie pas un changement dans l'esprit et dans les objectifs de la Société de médecine périnatale : celle-ci reste orientée vers un rôle de formation continue, multidisciplinaire ; mais elle bénéficiera ainsi de forces de propositions vives, car libérées d'un processus de décision, qui reste attribué au conseil d'administration. Un grand merci aux membres du CS, qui se sont tous mobilisés et ont travaillé très rapidement aux communications du congrès d'Angers, malgré une sollicitation pleinement estivale. L'ambiance de la première réunion a été à l'égal de celle connue à la SFMP : conviviale, pétillante, et les discussions ont été de haut niveau.

\footnotetext{
${ }^{1}$ La composition définitive du conseil scientifique est en cours de finalisation.
} 